\title{
Cultura organizacional y satisfacción laboral en la facultad de Ingeniería Industrial en el marco de la acreditación universitaria
}

\section{RESUMEN}

El mundo contemporáneo requiere de organizaciones e instituciones consolidadas, con capacidad para alcanzar adecuadamente su misión y visión, en donde el recurso humano tiene un rol fundamental.

Cada organización presenta singularidad en su cultura organizacional, la misma que puede ser gestionada en función del plan estratégico de la misma. Cabe mencionar que la cultura organizacional no se forma mediante discursos, sino mediante acciones sólidas.

De igual manera, el nivel de satisfacción de las personas que laboran en un institución, pública o privada, es un aspecto que no se soslaya en la administración moderna, en tanto depende de su acción el logro de los objetivos organizacionales.

El estudio abordó la relación entre cultura organizacional y satisfacción laboral en el estamento docente de la facultad de ingeniería industrial de la UNMSM, el mismo que contempló el reconocimiento del estado del arte relacionado al problema de investigación, la elaboración teórica de cada una de las variables, hasta arribar a sendos instrumentos de medición de los constructos. Se encontró una relación directa entre ambas variables.

Palabras clave: cultura organizacional, satisfacción laboral

ORGaNIZATIONAL CULTURE AND JOB SATISFACTION ON THE FACULTY OF INDUSTRIAL ENGINEERING IN THE CONTEXT OF UNIVERSITY ACCREDITATION

ABSTRACT

The contemporary world requires consolidated organizations and institutions with the capacity to adequately fulfill its mission and vision, in which human resource plays a crucial role.

Each organization has their uniqueness in organizational culture, it can be managed according to the strategic plan of the same. It is noteworthy that the organizational culture is not formed through speeches, but through strong actions.

Similarly, the level of satisfaction of the people who work at the institution, public or private, is an aspect not missed in modern management, while its action depends on the achievement of organizational objectives.

The study addressed the relationship between organizational culture and job satisfaction of teachers establishment of the Faculty of Mechanical Engineering of San Marcos, the same which looked upon the recognition of the state of research related to the problem of art, theoretical elaboration of each of the variables, arriving at their instruments measuring constructs. A direct relationship between the two variables was found.

Keywords: job satisfaction, organizational culture

\section{PLANTEAMIENTO DEL ESTUDIO}

El estudio del comportamiento individual y de grupo ha inquietado a diversos investigadores. Pettigrew, citado por Macintosh (2010), demostró que los conceptos de simbolismo, lenguaje y rituales pueden ser usados para analizar y comprender la vida organizacional.

Lacatus (2010), citando los trabajos de Flynn (2001) y Kusluva (2003), afirma que la cultura organizacional actúa como un sistema de control social y puede influir en las actitudes y el comportamiento de los empleados a través de los valores y creencias que operan en una empresa. La cultura organizacional ha demostrado tener influencia directa en la satisfacción del personal y el compromiso institucional.

Diaz (2003) esbozó un sistema descriptivo de los hechos sometidos a observación partiendo del supuesto básico de que en las universidades existen factores de clima y cultura organizacionales que influyen en el comportamiento de sus miembros como colectivo. Para sustentar el trabajo se revisaron diferentes aspectos de la Teoría de la Organización haciendo énfasis en los aspectos de Cultura y Clima, así como la Teoría de la Investigación. Concluye señalando haber encontrado una clara visión de la influencia que la Cultura organizacional (sistema de creencias, valores y normas) ejerce sobre el Clima Organizacional (tanto en su aspecto psicológico como físico) podría convertirse en una excelente herramienta para diagnosticar el estado de las organizaciones investigativas universitarias.

Bustamante (2008), en una investigación sobre el impacto de la implementación de mecanismos de control en una universidad colombiana, encontró que: los elementos del ambiente de control en general, no mostraron un comportamiento homogéneo desde el punto de vista de la cultura organizacional, hay un desconocimiento de los principios y valores de la Universidad por parte de los servidores públicos, se presentan inconformismos por ciertos manejos laborales, que se realizan en la organización desde el punto de vista del desarrollo del talento humano y se está generando una brecha entre la alta dirección y los empleados, pues estos últimos, han ido perdiendo la confianza y el compromiso directo hacia la organización.

* Docente Asociado FII. E-mail: otinocog@gmail.com

** Docente Principal FII. E-mail: cquispea9@gmail.com

*** Docente Asociado FII. E-mail: vbeltrans@hotmail.com 
Pelekais (2008) desarrolló una investigación para analizar la relación entre cultura organizacional y responsabilidad social en una universidad pública, concluyendo que los resultados encontrados evidencian que existe una relación directamente proporcional y moderada entre los elementos de la cultura y la responsabilidad social.

Calderón (2003) encontró, en un estudio realizado en la facultad de Ciencias Económicas de Bogotá que la satisfacción del trabajador universitario está asociado con culturas orientadas al empleado, corporativistas, pragmáticas y con control laxo.

Sanchez (2010), en su tesis doctoral sobre cultura organizacional en universidades mexicanas, concluye que en las instituciones universitarias incluidas en su investigación coexisten diferentes tipos de cultura, imperando la cultura en donde prima la participación y el compromiso, seguida de la cultura en donde predomina el control y la eficiencia. Se registra también un tipo de cultura donde predomina el mercado y la productividad.

Respecto a la satisfacción, se puede señalar que esta es la resultante de un proceso que se inicia en el sujeto concreto y real, y culmina en él mismo, estamos haciendo referencia a un fenómeno esencialmente subjetivo desde su naturaleza hasta la propia medición e interpretación de su presencia o no. La satisfacción entonces no estaría dada sólo como una sensación o estado individual y único, aunque esa es su esencia, sino que sería esa sensación o estado único e irrepetible que se produce en cada sujeto dado el desarrollo de todo un complejo proceso intra subjetivo e inter subjetivo. La satisfacción en el trabajo es la medida en que son satisfechas determinadas necesidades del trabajador y el grado en que éste ve realizadas las diferentes aspiraciones que puede tener en su trabajo, ya sean de tipo social, personal, económico o higiénico. Mediante los índices de satisfacción laboral normalmente se pretende auscultar a una población laboral para ver si tiene algún mal remediable o si todo marcha sobre ruedas. No es posible describir con cierto rigor las situaciones de trabajo sin tener en cuenta lo que dice el trabajador mismo (Cantera, 2004).

La mayor parte de los instrumentos de medición de la satisfacción laboral que se utilizan en la actualidad, interrogan sobre algunas dimensiones que se pueden aislar del siguiente modo (Cantera, 2004):

- El trabajo como tal (contenido, autonomía, interés, posibilidades de éxito).
- Relaciones humanas (estilo de mando; competencia y afabilidad de compañeros, jefes y subordinados).

- Organización del trabajo.

- Posibilidades de ascenso.

- Salario y otros tipos de recompensa.

Tal como señala Torres (2012), los sistemas de acreditación institucional, con el objetivo explícito de asegurar la calidad, la transparencia, la utilización eficaz de bienes públicos y privados, así como facilitar la comparabilidad en un mundo cambiante y globalizado, son una realidad en el ámbito universitario mundial $\mathrm{y}$, particularmente, latinoamericano.

Strydom et al (2004), profundizan, en relación a los impactos de la acreditación universitaria en Sudáfrica, en las relaciones y tensiones derivadas de los cambios que la implementación de estos sistemas trae aparejados en la cultura organizacional y las principales fuentes de resistencia en los estamentos docentes y administrativos, los mismos que dificultan el establecimiento de una cultura de calidad.

El modelo de acreditación planteado en el Perú por el CONEAU establece la importancia de la eficacia en el desarrollo de una cultura organizacional en la carrera y como ésta se relaciona con el grado de cumplimiento de las actividades planteadas para tal fin.

Los indicadores de gestión, GI-09, GI-13, Gl-14, abordan tanto el tema de cultura organizacional como los de satisfacción de docentes, estudiantes y trabajadores, con los programas de motivación e incentivos.

Tabash (2010), en un estudio sobre relación de la satisfacción laboral y cultura organizacional de trabajadores de una universidad costarricense, encontró hallazgos importantes:

- Aunque el estudio revela un porcentaje alto de satisfacción en los procesos comunicacionales, por lo estratégico que resulta el tema de la comunicación, es importante apuntar que una tercera parte de los sujetos de estudio manifiestan una opinión de poca o ninguna satisfacción.

- La comunicación en términos de información efectiva muestra el estudio que el $60 \%$ de los funcionarios están poco o nada satisfechos. Esto lleva a pensar que existen grados de distorsión en los procesos comunicacionales. 
- Por otra parte, un importante porcentaje (40\%) expresa insatisfacción con el trato personal y profesional recibido.

- Con respecto a oportunidades de progreso profesional existe más de la mitad de la población insatisfecha. No se percibe una verdadera oportunidad de crecimiento y desarrollo profesional.

- Salarialmente se encuentran satisfechos, no así con el reconocimiento al mérito. Existe una tendencia muy marcada con respecto a manifestar que el trabajo realizado no es evaluado en forma justa.

Silva (2009), en un estudio que relaciona satisfacción laboral y clima organizacional en la facultad de Medicina de la UNAP (Perú), concluye que se obtuvo como resultados que dos tercios del personal docente mostraban insatisfacción en su centro laboral y sensación de frustración; dos tercios del personal administrativo mostraron satisfacción laboral.

En cuanto al clima laboral cerca del $70 \%$ del personal tanto administrativo como docente lo calificaron como positivo.

En base a estos antecedentes señalados el estudio se orientó a determinar la relación entre la Cultura Organizacional y la Satisfacción Laboral del personal docente que labora en la facultad de Ingeniería Industrial de la UNMSM. Para establecer la relación entre las dos variables, cultura organizacional y satisfacción laboral, se elaboraron sendos instrumentos de medición (ver anexo), en base al marco teórico elaborado, los mismos que se sub dividieron en dimensiones, cuya relación se muestra en el cuadro 1

\section{MARCO TEÓRICO}

El concepto subyacente para el presente estudio es el de cultura. El primero que abordó una interpretación sociológica y antropológica de este concepto fue Edwars Tylor (1871), quien la define como un todo complejo que incluye el conocimiento, las creencias, el arte, el derecho, la moral, las costumbres y cualquier otra capacidad y hábitos adquiridos por el hombre como miembro de la sociedad.

La cultura es el pegamento social o normativo que mantiene unida a una organización. Expresa los valores o ideales sociales y creencias que los miembros de la organización llegan a compartir, manifestadas en elementos simbólicos, como mitos, rituales, historias, leyendas y un lenguaje especializado.
Cuadro 1. Dimensiones de las variables en estudio

\begin{tabular}{|c|c|}
\hline $\begin{array}{c}\text { CULTURA } \\
\text { ORGANIZACIONAL }\end{array}$ & SATISFACCIÓN LABORAL \\
\hline $\begin{array}{l}\text { Motivación: } 1 \text { - } 5 \\
\text { Valores: } 6 \text { - } 11 \\
\text { Comunicación: } 12 \text { - } 16 \\
\text { Identificación: 17-20 }\end{array}$ & $\begin{array}{l}\text { F1. Desarrollo profesional } \\
(1-4) \\
\text { F2. Relación con el jefe } \\
(5-8) \\
\text { F3. Relación con } \\
\text { compañeros (9-12) } \\
\text { F4. Desarrollo de la función } \\
\text { (13-17) } \\
\text { F5. Permanencia (18-20) } \\
\text { F6. Condiciones físicas } \\
\text { (21-22) }\end{array}$ \\
\hline
\end{tabular}

Fuente: Elaboración propia

Según Hofstede (2001), citado por Macintosh (2010), cultura presupone una colectividad, determina la unidad de un grupo. Organizaciones, a su vez, son entidades simbólicas, en tanto funcionan con modelos implícitos en las cabezas de sus miembros, y esos modelos son culturalmente determinados.

Larentis (2010), siguiendo a Freitas (2002), señala acertadamente que no se pueden concebir organizaciones independientes del contexto y de la época en que se sitúan. Añade Larentis que la forma como las organizaciones son estructuradas indica que las personas son, en grados diferentes, dependientes entre sí para conseguir que las cosas sean bien hechas.

Cavedon (2003) entiende la cultura organizacional como una red de significaciones circulando dentro y fuera del espacio organizacional, siendo simultáneamente ambiguas, contradictorias, complementarias, dispares, análoga, lo que conlleva a considerar tanto homogeneidades como heterogeneidades en una organización.

Larentis (2010) señala que para comprender la cultura de una organización se deben tener en cuenta los artefactos, las creaciones, la arquitectura, el diseño, los modelos de comportamiento visibles e invisibles, la manera como se visten las personas, el nivel de los valores manifiestos y el nivel de los presupuestos inconscientes.

Cultura organizacional es el modo de vida propio de cada organización que se desarrolla en cada uno de sus miembros. La cultura organizacional es un 
conjunto de valores, creencias, tradiciones y modos de ejecutar las tareas que de manera consciente o inconsciente, cada organización adopta y acumula con el tiempo y que condiciona fuertemente el pensamiento y el comportamiento de sus miembros. Esta se enraíza en la historia de cada organización, en sus éxitos y fracasos.

Al respecto, Libreros (2011) señala que los principales elementos no visibles de una cultura organizacional son los valores. Los valores de una organización, constituyen un tipo especial de creencias compartidas por sus miembros, quienes determinan el deber ser, es decir, lo que es bueno y deseable (valores positivos) y lo que es malo y no deseable (valores negativos).

Marcano (2011) resalta la conveniencia de que cada organización conozca su cultura, ya que, le permite en gran medida describir su propia personalidad, que la hace distinta de las demás. Además, permite la ayuda necesaria para definir, analizar y resolver aspectos que tienen que ver con la identidad de los miembros de la organización. Sirve también como un mecanismo de control de guía y moldea las actitudes y el comportamiento de los trabajadores.

Lezama (2008) establece que las dimensiones de la cultura organizacional son seis: Mitos e Ideologías, Valores, Estructura Organizacional, Sistemas Motivacionales, Sentido de Pertenencia y Procesos de Cambio y Comunicación.

Agustini et al (2012), citan un estudio de Añez (2006), según el cual las dimensiones a tener en cuenta son: la Identificación Organizacional, el Grado de Compromiso y Pertenencia hacia las Instituciones, así como la Autonomía con la cual los individuos desarrollan sus actividades y decisiones, el nivel de apoyo recibido para ejecutar y llevar a efecto sus labores, enmarcado significativamente por el estilo gerencial adoptado por los miembros directrices de la organización, donde también se deben tomar en cuenta el manejo del trabajo en equipo, los cambios continuos y la tolerancia de los trabajadores ante la presencia de los conflictos presentados en la organización.

El estudio de las condiciones socio laborales ha adquirido importancia en los últimos años y ahora la satisfacción laboral se considera un constructo muy importante en psicología organizacional y del trabajo, y en el ámbito de las investigaciones educativas, por el papel mediador que juega entre las condiciones del entorno laboral y las consecuencias para el desarrollo de la institución tanto organizacional como individual (Dorman \& Zapf, 2001).
La satisfacción con respecto al trabajo deviene del contacto de las personas con los puestos de trabajo en organizaciones laborales (públicas o privadas) y constituye expresión del sentimiento que experimenta el trabajador como consecuencia del grado en que percibe la acción de la organización en la que actúa.

La teoria de los factores motivacionales en relación a la satisfacción laboral fue presentada por Herzberg (citado por Davis, 2004, p. 127) y explica el comportamiento de las personas en situaciones de trabajo, y los llamó factores de higiene o mantenimiento (extrínsecos) y factores motivacionales (intrínsecos).

Para López (2005), la satisfacción laboral implica una actitud, o más bien un conjunto de actitudes y una tendencia valorativa de los individuos y los colectivos en el contexto laboral que influirán de una manera significativa en los comportamientos y desde luego en los resultados. Añade este investigador que la satisfacción laboral también puede ser considerada como la correspondencia entre las demandas de los individuos (expresada en necesidades y motivos) y lo que percibe que la organización propicia y le otorga de una manera u otra.

En la búsqueda de una percepción integral de la satisfacción laboral, López (2005) propone y valida un instrumento alternativo para medir la satisfacción laboral. Con esta concepción los grupos o factores que serán considerados están vinculados a:

- La naturaleza y contenido de trabajo.

- El trabajo en grupo y sus directivos

- Las condiciones de trabajo

- El esfuerzo y los resultados individuales.

- Las condiciones de bienestar.

\section{MATERIALES Y MÉTODOS}

Se desarrolló una investigación aplicada con diseño descriptivo-correlacional, tomando como base la población de docentes de la facultad de Ingeniería Industrial de la UNMSM. Se delimitó la participación de docentes nombrados.

Tomando como referencia el trabajo de López, se adaptó el instrumento de medición de satisfacción laboral al ámbito de los docentes de instituciones universitarias, considerando cuatro dimensiones y 22 ítems. Las dimensiones consideradas se presentan en el cuadro 1. 
Siguiendo a Agustini et al (2012), en el cuadro 1 se consideraron cuatro dimensiones de la cultura organizacional. Ver cuadro 1.

\section{RESULTADOS}

Tomando como referencia un total de 60 docentes nombrados en la facultad de Ingeniería Industrial, el estudio comprendió a 58 de ellos (Cuadro 2).

La mayoría de los docentes tienen más de 10 años en tiempo de servicios a la universidad, perteneciendo la mayoría de ellos al departamento académico de Diseño y Tecnología Industrial.

Según el cuadro 3 , la mayoría de los docentes $(62,1 \%)$ tienen más de 50 años, siendo esa proporción más marcada en los departamentos de Diseño y Producción.

Según el cuadro 4, las puntuaciones promedio de los docentes denotan un índice de satisfacción laboral de 75.76 sobre un total de 110 puntos (69\%).

En la dimensión desarrollo profesional alcanzan en promedio 15.10 sobre un total de 20 puntos (76\%); en la relación con su jefe obtienen 15.34 $(77 \%)$; en su relación con sus colegas alcanzan 12.95 (65\%); mientras que en el desarrollo de sus funciones obtienen 16.02 (64\%); alcanzan 9.71 $(65 \%)$ en permanencia; finalmente, en el aspecto de las condiciones físicas para laborar obtienen una puntuación promedio de 6.79 (68\%).

Cuadro 2. Tiempo de servicios según Departamento Académico.

\begin{tabular}{|l|c|c|c|c|c|}
\hline \multirow{2}{*}{\multicolumn{2}{|c|}{ Departamento }} & \multicolumn{3}{c|}{ Tiempo de servicio (años) } & \multirow{2}{*}{ Total } \\
\cline { 2 - 5 } & Hasta 2 & $\mathbf{3 - 5}$ & $\mathbf{6 - 1 0}$ & $\mathbf{> 1 0}$ & \\
\hline Sistemas e informática & & & $5.2 \%$ & $25.9 \%$ & $31.0 \%$ \\
\hline Diseño y tecnología industrial & $5.2 \%$ & $10.3 \%$ & $3.4 \%$ & $20.7 \%$ & $39.7 \%$ \\
\hline Producción y gestión industrial & & $10.3 \%$ & $5.2 \%$ & $13.8 \%$ & $29.3 \%$ \\
\hline Total & $5.2 \%$ & $20.7 \%$ & $13.8 \%$ & $60.3 \%$ & $100.0 \%$ \\
\hline
\end{tabular}

Cuadro 3. Tiempo de servicios según Edad.

\begin{tabular}{|c|c|c|c|}
\hline \multirow{2}{*}{ Departamento } & \multicolumn{2}{|c|}{ Tiempo de servicio (años) } & \multirow{2}{*}{ Total } \\
\hline & De 30 a 50 años & Mayor de 50 años & \\
\hline Sistemas e informática & $13.8 \%$ & $17.2 \%$ & $31.0 \%$ \\
\hline Diseño y tecnología industrial & $17.2 \%$ & $22.4 \%$ & $39.7 \%$ \\
\hline Producción y gestión industrial & $6.9 \%$ & $22.4 \%$ & $29.3 \%$ \\
\hline Total & $37.9 \%$ & $62.1 \%$ & $100.0 \%$ \\
\hline
\end{tabular}

Cuadro 4. Estadísticos descriptivos variable Satisfacción Laboral.

\begin{tabular}{|l|c|c|c|c|c|}
\hline & Mínimo & Máximo & Media & Desv. típ. & Escala \\
\hline Satisfacción Laboral & 39 & 93 & 75.76 & 10.67 & $22-110$ \\
\hline Dimensiones & \multicolumn{5}{|l}{} \\
\hline Desarrollo profesional & 6 & 20 & 15.10 & 2.51 & $4-20$ \\
\hline Relación con el Jefe & 4 & 20 & 15.34 & 2.46 & $4-20$ \\
\hline Relación con los colegas & 8 & 20 & 12.95 & 2.90 & $4-20$ \\
\hline Desarrollo funciones & 11 & 25 & 16.02 & 3.08 & $5-25$ \\
\hline Permanencia & 3 & 15 & 9.71 & 2.45 & $3-15$ \\
\hline Condiciones físicas & 2 & 10 & 6.79 & 2.00 & $2-10$ \\
\hline
\end{tabular}


En todas las dimensiones de la variable satisfacción laboral se obtuvieron puntuaciones que denotan un adecuado índice de satisfacción laboral, destacando las dimensiones de desarrollo profesional y relación con el jefe del departamento académico.

Conforme al cuadro 5 , la puntuación promedio obtenida por los docentes en la variable cultura organizacional fue de 60.03 , sobre un máximo de 100 , lo cual indica un índice del $60 \%$, que implica un nivel aceptable de la cultura organizacional de los docentes en la facultad de Ingeniería Industrial.

En el aspecto de motivación, el puntaje promedio fue de 15.33 sobre un máximo de 25 puntos (61\%); en la dimensión de valores que se cultivan en la FII el promedio alcanzado fue de 17.55 sobre un total de 30 puntos (59\%); en los niveles de comunicación al interior de la facultad se obtiene 15.84 sobre un total de 25 puntos (63\%); finalmente, en el aspecto de identificación institucional, el puntaje promedio obtenido fue de 11.09 sobre un total de 20 puntos (55\%).
En el cuadro 6 se muestran los puntajes para ambas variables según cada departamento académico, cuyos resultados denotan diferencias en ambos aspectos. Se ilustran las diferencias de las características de cultura organizacional entre los docentes de los tres departamentos académicos, así como de los niveles de satisfacción laboral. Se aprecia que en el departamento de Diseño y Tecnología Industrial los promedios obtenidos son mayores que el promedio general.

Con la finalidad de analizar la relación entre cultura organizacional y satisfacción laboral, en los docentes de la facultad de Ingeniería Industrial, se aplicó la prueba de correlación de Spearman, a partir de los puntajes totales obtenidos para cada variable.

Como se ilustra en el cuadro 7 , El coeficiente de correlación de Spearman obtenido fue de 0.545, que denota una relación positiva débil entre ambas variables.

Cuadro 5. Estadísticos descriptivos de la variable Cultura Organizacional.

\begin{tabular}{|l|c|c|c|c|c|}
\hline & Mínimo & Máximo & Media & Desv. típ. & Escala \\
\hline Cultura Organizacional & 20 & 88 & 60.03 & 11.72 & $20-100$ \\
\hline & \multicolumn{5}{|l}{} \\
\hline Motivación & 5 & 25 & 15.33 & 3.83 & $5-25$ \\
\hline Valores & 6 & 30 & 17.55 & 3.92 & $6-30$ \\
\hline Comunicación & 5 & 23 & 15.84 & 3.35 & $5-25$ \\
\hline Identificación & 4 & 16 & 11.09 & 2.73 & $4-20$ \\
\hline
\end{tabular}

Cuadro 6. Puntajes según departamento académico.

\begin{tabular}{|c|l|c|c|c|c|}
\hline \multicolumn{1}{|c|}{ Departamento } & & N & Media & General & Máximo \\
\hline \multirow{2}{*}{ Sistema e informática } & Satisfacción Laboral & 18 & 74.89 & 75.76 & 110 \\
\cline { 2 - 6 } & Cultura Organizacional & 18 & 59.22 & 60.03 & 100 \\
\hline \multirow{2}{*}{ Diseño y tecnología industrial } & Satisfacción Laboral & 23 & 77.22 & 75.76 & 110 \\
\cline { 2 - 6 } & Cultura Organizacional & 23 & 62.78 & 60.03 & 100 \\
\hline \multirow{2}{*}{ Producción y gestión industrial } & Satisfacción Laboral & 17 & 74.71 & 75.76 & 110 \\
\cline { 2 - 6 } & Cultura Organizacional & 17 & 57.18 & 60.03 & 100 \\
\hline
\end{tabular}

Cuadro 7. Correlación entre las variables.

\begin{tabular}{|l|c|c|}
\hline & Cultura Organizacional & Satisfacción Laboral \\
\hline Cultura Organizacional & 1 &, $545^{* *}$ \\
\hline Satisfacción Laboral &, $545^{* *}$ & 1 \\
\hline
\end{tabular}


Cuadro 8. Correlaciones según departamento académico.

\begin{tabular}{|l|l|c|c|}
\hline \multicolumn{2}{|l|}{ Departamento académico } & $\begin{array}{c}\text { Cultura } \\
\text { Organizacional }\end{array}$ & $\begin{array}{c}\text { Satisfacción } \\
\text { Laboral }\end{array}$ \\
\hline \multirow{2}{*}{ Sistema e Informática } & Cultura Organizacional & 1 &, $534^{*}$ \\
\cline { 2 - 4 } & Satisfacción Laboral &, $534^{*}$ & 1 \\
\hline \multirow{2}{*}{ Diseño y Tecnología Industrial } & Cultura Organizacional & 1 & .356 \\
\cline { 2 - 4 } & Satisfacción Laboral & .356 & 1 \\
\hline \multirow{2}{*}{ Producción y Gestión Industrial } & Cultura Organizacional & 1 & 1 \\
\cline { 2 - 4 } & Satisfacción Laboral &, $741^{* *}$ \\
\hline
\end{tabular}

En el cuadro 8 se muestran las correlaciones obtenidas para ambas variables, de acuerdo al departamento académico.

En el departamento de Sistemas e Informática, la correlación observada entre cultura organizacional y satisfacción laboral es positiva y regular $($ rho $=0.534)$. En el caso de los docentes de Diseño y Tecnología Industrial la correlación observada es positiva y débil (rho $=0.356$ ); mientras que en el departamento de Producción y Gestión Industrial la asociación es positiva y regular (rho $=0.741$ )

Los cuadros 7 y 8 han permitido verificar la hipótesis de investigación. La correlación positiva, de 0,545, entre cultura organizacional y satisfacción laboral denota una asociación positiva entre ambas variables. Una mejor cultura organizacional conduce a mejores niveles de satisfacción laboral entre los docentes de la facultad de ingeniería industrial. Se confirman de esta manera los resultados obtenidos por Silva.

\section{CONCLUSIONES Y RECOMENDACIONES}

Tanto la cultura organizacional como la satisfacción laboral de los docentes presentan un índice aceptable, el mismo que debe ser mejorado.

Se ha demostrado que existe una relación positiva, aunque débil, entre la cultura organizacional y la satisfacción laboral en docentes de la facultad de Ingeniería Industrial de la UNMSM, asociación que permite afirmar que si se mejoran los aspectos de motivación, identificación institucional, comunicación y se fortalecen los valores, se obtendrán mejores niveles de satisfacción laboral.

Esta asociación no es uniforme en los tres departamentos académicos, asi como no son uniformes las manifestaciones de cultura organizacional ni satisfacción laboral, aspecto que debe ser tomado en cuenta por la gestión de la facultad de Ingeniería Industrial.

En la cultura organizacional, las dimensiones con mejor resultado corresponden a comunicación y motivación; siendo menores en los aspectos relacionados a los valores y a la identificación institucional.

Respecto a la satisfacción laboral, los mejores índices se presentan en las dimensiones de desarrollo profesional y relación con su jefe. Se observan menores puntuaciones en la permanencia y en el desarrollo de sus funciones.

Se recomienda implementar un conjunto de estrategias que permitan mejorar las características tanto de cultura organizacional como satisfacción laboral, evaluadas en cada una de sus dimensiones.

\section{REFERENCIAS BIBLIOGRÁFICAS}

[1] Agustini et al. (2012). La cultura organizacional. Un enfoque de casos. UNMSM. Lima.

[2] Bustamante, Jonatan (2008). Impacto en la cultura organizacional con la aplicación del modelo estandar de control interno (meci) en la Universidad de Antioquia. Colombia.

[3] Calderón, Gregorio (2008). Cultura organizacional y bienestar laboral en una institución universitaria. Revista Cuadernos de administración. Vol $16 \mathrm{~N}^{\circ} 25$. Bogotá. Colombia.

[4] Cantera, F. (2004). NTP 212: Evaluación de la satisfacción laboral: métodos directos e indirectos. Ministerios de Trabajo y asuntos sociales. España. Disponible en http://www. insht.es/InshtWeb/Contenidos/Documentacion/ FichasTecnicas/NTP/Ficheros/201a300/ ntp_212.pdf. Consultado Noviembre 2013. 
[5] Cavedon, Neusa (2003). Antropología para Administradores. Universidad Estatal Porto Alegre. Brasil.

[6] Dormann \& Zapf (2001). Job satisfaction: a meta-analysis of stabilities", Journal of Organizational Behavior, vol. 22.

[7] Diaz, Alejandra (2003). Investigación universitaria, clima y cultura organizacional. Tesis doctoral. Universidad Nacional Experimental Simón Rodriguez (Venezuela).

[8] Lacatus, Mariana (2013). Organizacional culture in contemporary university. Bucharest University of Economic Studies.

[9] Larentis, fabiano (2010). Marketing relacional y cultura organizacional. Tesis doctoral. Universidad federal de Rio Grande del Sur. Brasil.

[10] Lezama, Lucila (2008). El sistema cultural y sociocultural en la cultura organizacional. Revista electrónica de psicología Universidad Veracruz. México. Consultado en: http://www. uv.mx/psicologia/revista-electronica-procesospsicologicos-y-sociales/revistas/vol-4-ano2008-no-1-y-2/. Agosto 2013.

[11] Libreros, Andrés. (2011). Incidencia de la cultura organizacional sobre el bienestar laboral de los servidores publicos de una institución educativa del Valle del Cauca. Tesis de Maestría. Universidad Nacional de Colombia.

[12] Macintosh, Erick \& Doherty, Alison (2010). The influence of organizacional culture on job satisfaction and intention to leave. Sport Management Review 13 (106 - 117).

[13] Marcano. Ricardo. (2010). La cultura organizacional del personal docente de la
Universidad de Oriente, Núcleo de Sucre. Tesis de Licenciatura en Administración.

[14] Murillo, Milena et al (2003). Cultura organizacional y bienestar laboral. Revista Cuadernos de Investigación. Volumen 16, N. ${ }^{\circ}$ 025. Pontificia Universidad Javeriana. Bogotá.

[15] Pelekais, Clara (2008). Cultura organizacional y la responsabilidad social en las universidades públicas. Revista Ciencias Sociales, vol XIV N ${ }^{\circ}$ 1. Universidad de Zulia. Venezuela.

[16] Sanchez, Mirna (2010).Caracterización de la Cultura Organizacional de instituciones de educación superior del Estado de Guanajato. Tesis doctoral. Universidad Autónoma de Querétaro. México.

[17] Silva Delgado, H (2009). "Medición del clima laboral y satisfacción del personal de trabajadores docentes y no docentes de la facultad de Medicina UNAP-2009. http:// www.unapiquitos.edu.pe/oficinas/iiunap/ articulos2009.html. Revisado 12/12/2013.

[19] Strydom, J., Z, N. y Murria, L. (2004): Quality, culture and change. Quality in higher EducaUJPn, Vol.10, №3.

[20] Tabash, Alexandra (2010). Factores de la Cultura Organizacional influyentes en la satisfacción laboral del personal administrativo de la Sede Regional Brunca, Campus Pérez Zeledón Universidad Nacional Costa Rica.

[21] Torres, Emilio (2012). Acreditación institucional y la Mirada de los actors. Un estudio cualitativo en universidades privadas de Santiago. Revista Estudios Pedagógicos XXXVIII, N. ${ }^{\circ}$ 2: 221-242, 2012. Universidad Austral de Chile. 


\section{ANEXOS}

\section{CUESTIONARIO DE CULTURA ORGANIZACIONAL}

Marque con una $\mathbf{X}$ la casilla que MÁS COINCIDA CON SU PUNTO DE VISTA respecto a los siguientes enunciados; considerando los niveles de la tabla que sigue:

\begin{tabular}{|c|c|c|c|c|}
\hline TA & A & I & $\mathrm{D}$ & TD \\
\hline $\begin{array}{l}\text { Totalmente de } \\
\text { Acuerdo }\end{array}$ & De Acuerdo & Indiferente & En Desacuerdo & $\begin{array}{c}\text { Totalmente en } \\
\text { Desacuerdo }\end{array}$ \\
\hline
\end{tabular}

\begin{tabular}{|c|c|c|c|c|c|c|}
\hline $\mathbf{N}^{\circ}$ & ENUNCIADO & TA & A & I & D & TD \\
\hline 1 & $\begin{array}{l}\text { En la facultad existe una verdadera preocupación por las condiciones } \\
\text { de trabajo de todos los docentes }\end{array}$ & & & & & \\
\hline 2 & $\begin{array}{l}\text { Los directivos motivan constantemente el buen desempeño de los } \\
\text { docentes }\end{array}$ & & & & & \\
\hline 3 & $\begin{array}{l}\text { Lo que nos diferencia de otras facultades es el entusiasmo que ponemos } \\
\text { en el logro de los objetivos institucionales. }\end{array}$ & & & & & \\
\hline 4 & $\begin{array}{l}\text { En esta facultad se ofrece un ambiente que estimula a los docentes y } \\
\text { alumnos a dar lo mejor de sí y se acostumbra premiar los logros por } \\
\text { muy pequeños que estos sean. }\end{array}$ & & & & & \\
\hline 5 & $\begin{array}{l}\text { En esta facultad se acostumbra a reconocer los esfuerzos de los } \\
\text { docentes y existe un justo reconocimiento. }\end{array}$ & & & & & \\
\hline 6 & $\begin{array}{l}\text { La credibilidad de los directivos se ha sustentado, a través del tiempo, } \\
\text { en la coherencia que han logrado establecer entre lo que dicen y hacen. }\end{array}$ & & & & & \\
\hline 7 & En esta facultad se reconoce la puntualidad de todos los docentes. & & & & & \\
\hline 8 & $\begin{array}{l}\text { En esta facultad, al asignar los recursos materiales, siempre se } \\
\text { ha procurado hacerlo con equidad y, al culminar un proyecto, se } \\
\text { acostumbra a presentar el balance correspondiente. }\end{array}$ & & & & & \\
\hline 9 & $\begin{array}{l}\text { En esta facultad existe respeto mutuo entre los actores educativos y } \\
\text { hay una secuencia de hechos que demuestran una estrecha unión y } \\
\text { camaradería.. }\end{array}$ & & & & & \\
\hline 10 & $\begin{array}{l}\text { Todos los docentes tienen especial cuidado en preservar la buena } \\
\text { imagen de la facultad. }\end{array}$ & & & & & \\
\hline 11 & $\begin{array}{l}\text { La existencia de canales de comunicación, ágiles y expeditos, } \\
\text { garantizan el éxito de nuestro trabajo educativo. }\end{array}$ & & & & & \\
\hline 12 & $\begin{array}{l}\text { Se trabaja por mantener una comunicación que facilite la integración y } \\
\text { cohesión entre todos los docentes. }\end{array}$ & & & & & \\
\hline 13 & $\begin{array}{l}\text { En esta facultad acostumbramos a decirnos las cosas en forma clara } \\
\text { y directa. }\end{array}$ & & & & & \\
\hline 14 & $\begin{array}{l}\text { Tenemos un archivo (documentos, fotos, videos, etc) que nos recuerdan } \\
\text { las diversas etapas de desarrollo por la que hemos atravesado. }\end{array}$ & & & & & \\
\hline 15 & $\begin{array}{l}\text { Existe buena relación, comunicación y apoyo entre trabajadores } \\
\text { docentes y no docentes }\end{array}$ & & & & & \\
\hline 16 & $\begin{array}{l}\text { Existen buenas referencias sobre cómo, gracias al esfuerzo conjunto, } \\
\text { se lograron alcanzar metas específicas. }\end{array}$ & & & & & \\
\hline 17 & $\begin{array}{l}\text { Al iniciar cada período educativo, se revisan las metas planificadas y se } \\
\text { determinan sus logros y qué nos falta por alcanzar. }\end{array}$ & & & & & \\
\hline 18 & $\begin{array}{l}\text { Los cambios experimentados en esta facultad, desde su creación, nos } \\
\text { demuestran una vida creativa e innovadora. }\end{array}$ & & & & & \\
\hline
\end{tabular}




\begin{tabular}{|c|l|l|l|l|l|}
\hline 19 & $\begin{array}{l}\text { En esta facultad la participación es voluntaria para el desarrollo de } \\
\text { actividades y para el logro de los objetivos institucionales }\end{array}$ & & & & \\
\hline 20 & $\begin{array}{l}\text { Los docentes y trabajadores no docentes se identifican plenamente con } \\
\text { la facultad, lo que se comprueba en la vida cotidiana y en su entorno }\end{array}$ & & & & \\
\hline
\end{tabular}

\begin{tabular}{|c|c|c|c|c|}
\hline $\begin{array}{c}\text { TA } \\
\begin{array}{c}\text { Totalmente de } \\
\text { Acuerdo }\end{array}\end{array}$ & De Acuerdo & Indiferente & En Desacuerdo & $\begin{array}{c}\text { TD } \\
\text { Totalmente en } \\
\text { Desacuerdo }\end{array}$ \\
\hline
\end{tabular}

\section{Gracias por su colaboración}

\section{SATISFACCIÓN LABORAL}

Estimado(a) colaborador(a):

Con el propósito de conocer la satisfacción laboral en la facultad en donde usted labora agradeceremos su colaboración, marcando las respuestas a las preguntas correspondientes.

\section{CUESTIONARIO}

Marque con una $\mathrm{X}$ la casilla que más coincida con su opinión respecto a las siguientes afirmaciones, considerando los valores de la tabla siguiente:

Considere que:

\begin{tabular}{|c|c|c|c|c|}
\hline 1 & 2 & 3 & 4 & 5 \\
NUNCA & CASI NUNCA & A VECES & CASI SIEMPRE & SIEMPRE \\
\hline
\end{tabular}

\begin{tabular}{|c|c|c|c|c|c|c|}
\hline $\mathbf{N} .^{\circ}$ & AFIRMACIONES & 1 & 2 & 3 & 4 & 5 \\
\hline 1 & Se aprecia fácilmente el resultado de su trabajo en el producto final & & & & & \\
\hline 2 & $\begin{array}{l}\text { Su trabajo requiere realizar diferentes operaciones y utilizar un gran } \\
\text { número de habilidades y conocimientos }\end{array}$ & & & & & \\
\hline 3 & $\begin{array}{l}\text { Los resultados de su trabajo afectan significativamente la vida o bienestar } \\
\text { de otras personas }\end{array}$ & & & & & \\
\hline 4 & $\begin{array}{l}\text { Su propio trabajo, los supervisores u otras personas, le dan a conocer lo } \\
\text { bien que está desarrollando su labor }\end{array}$ & & & & & \\
\hline 5 & $\begin{array}{l}\text { Puede cada miembro del grupo desarrollar con habilidad todas o gran } \\
\text { parte de las tareas que tiene asignado el grupo }\end{array}$ & & & & & \\
\hline 6 & El grupo puede ver el resultado del trabajo en el producto final & & & & & \\
\hline 7 & $\begin{array}{l}\text { Los miembros del grupo, participan en la determinación de metas y } \\
\text { objetivos de trabajo }\end{array}$ & & & & & \\
\hline 8 & $\begin{array}{l}\text { El grupo, se mantiene unido para alcanzar una meta común después de } \\
\text { acordada }\end{array}$ & & & & & \\
\hline 9 & Se siente usted en todo momento apoyado por el grupo & & & & & \\
\hline 10 & $\begin{array}{l}\text { Está satisfecho con las relaciones humanas que existen entre los } \\
\text { miembros del grupo y de estos con sus directivos }\end{array}$ & & & & & \\
\hline
\end{tabular}




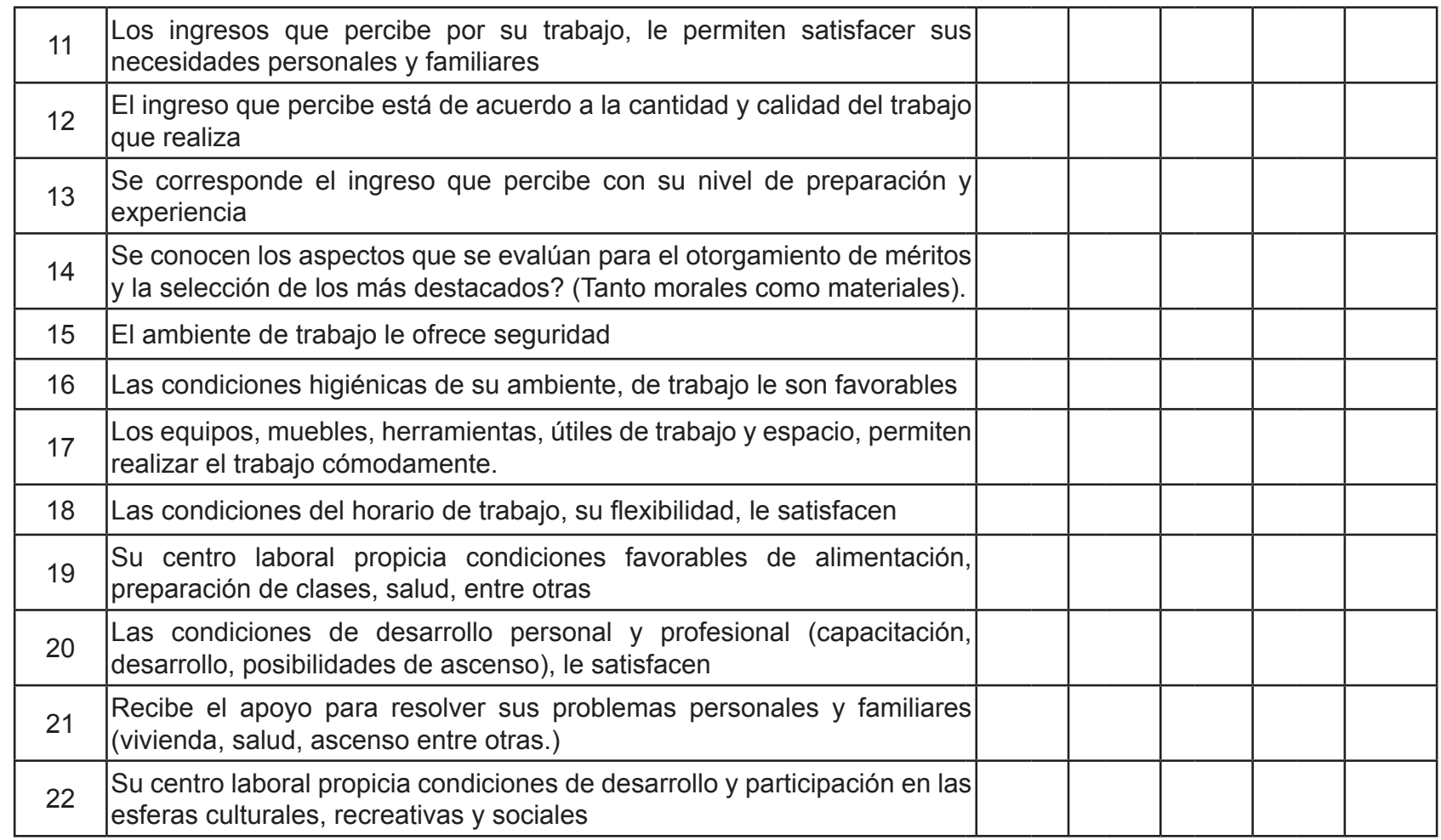

